\title{
HISTÓRIA EM CENA: A EXPERIÊNCIA DE CRIAÇÃO EM AUDIOVISUAL E SUAS TECNOLOGIAS NA REARTICULAÇÃO DO ENSINO DE HISTÓRIA
}

\author{
HISTORY ON SCENE: EXPERIENCING CREATION ON AUDIO-VISUAL AND ITS \\ TECHNOLOGIES ON REARTICULATING HISTORY TEACHING
}

\author{
Mário Luiz da Costa Assunção Júnior ${ }^{1}$
}

\begin{abstract}
RESUMO
O presente trabalho representa a proposta de pesquisa apresentada ao Programa de Pós-Graduação em Educação da Universidade Federal do Triângulo Mineiro - UFTM. Vinculada à Linha de pesquisa formação deprofessores e cultura digital, debate a utilização da linguagem audiovisual na rearticulação do ensino de História por meio da produção de curtas metragens em sala de aula. Partindo de um levantamento bibliográfico que evidencia a relevância do tema, a pesquisa realizará um estudo de duas experiências de produção de curtas metragens na realidade escolar. Os envolvidos nas produções se constituirão como sujeitos da pesquisa, sendo suas visões e experiências sistematizadas por meio de grupos focais e entrevistas. Pretende-se com tais procedimentos evidenciar a viabilidade da introdução da produção audiovisual em sala de aula e a permanência das experiências daqueles que participaram de tais produções.
\end{abstract}

PALAVRAS-CHAVE: Audiovisual. Curta-Metragem. Ensino de História. Ensino Médio.

\begin{abstract}
This paper represents the research proposal presented to the Post Graduation Program in Education of Federal University of Triangulo Mineiro - UFTM. Attached to the research line teaching formation and digital culture, it discusses the usage of audiovisual language on rearticulation of history teaching exploring short films making in classroom. After an alysing the literature about the relevancy of the theme, two short film production experiences on scholar reality will be studied. The ones involved on the production will be come research subjects and their experiences and points of view will be systematized using focal groups and interviews. Through such procedures, the purpose of the research is to show the viability to introduce audiovisual production in class and the maintainance of the experience from those who participated on the productions.
\end{abstract}

KEYWORDS: AudiovisuaL. Short film. History Teaching. High School.

\section{INTRODUÇÃO}

A coisa mais importante, em um filme, é o ponto de vista. Quando vejo os dez ou quinze primeiros planos de um longa-metragem, sei o que o diretor tem na cabeça - sei, sobretudo se ele tem algo na cabeça. É a visão de mundo dele que me interessa. O resto é cenário.

Oliver Stone

\footnotetext{
${ }^{1}$ Instituto Federal do Triângulo Mineiro - IFTM. Programa de Pós-Graduação Mestrado em Educação da Universidade Federal do Triângulo Mineiro - UFTM. Email: marioassuncao@iftm.edu.br
} 
O desafio da educação reside na reinvenção. O mundo constantemente se reorganiza em suas estruturas e significados exigindo daqueles que são agentes/sujeitos do processo de ensino uma reconfiguração de seus "sistemas". Esse ensinar que se reconfigura não pode, contudo, perder a beleza e a vida inerentes ao ato de educar (JESUS, 2011). A evolução dos sistemas de informação, articulados com as novas mídias e redes sociais, apresenta uma nova realidade para o universo docente, tendo em vista que os meios de tecnologia não são mais objeto de uso exclusivo por parte do professor e os estudantes não mais absorvem a tecnologia de forma passiva. É a incorporação das mídias na experiência cotidiana em todas as suas vertentes sendo que crianças e jovens passam a ser produtores de conteúdo (PIMENTA \& PRATA-LINHARES, 2013).

Vivemos uma era de revolução digital em que os impactos das novas tecnologias na vida cotidiana não encontram precedentes na história. Uma revolução tecnológica recria a realidade apontando novos caminhos possíveis e quebrando velhos paradigmas. Um novo panorama repleto de possibilidades, de ampliação do potencial humano e de construção de novos desafios (GABRIEL, 2013).

Observando tal realidade, percebemos que mais do que o simples acesso à tecnologia e à informação, é necessário transformá-las em conhecimento, libertando estudantes e professores da alienação. Para tanto, faz-se necessário um processo de incorporação crítica dessas novas ferramentas pelos docentes no universo da escola, pois o professor já não é mais um "provedor de conteúdos", mas sim um provocador de reflexões e conexões desse ambiente complexo e poderoso (Gabriel, 2013). Uma alfabetização midiática que permita a evolução ampla dos sujeitos do conhecimento dentro e fora da sala de aula (PIMENTA \& PRATALINHARES, 2013).

A cultura digital se propaga incessantemente determinando mudanças no processo de formação dos docentes e estudantes e na maneira como se relacionam com a produção e difusão do conhecimento. Dessa forma, "novos desafios se avizinham quanto à formação dos professores, sobretudo em relação às mudanças curriculares e didáticas e às novas diretrizes para as licenciaturas."

Nesse sentido, nenhuma mídia dialoga mais com a contemporaneidade no campo educacional do que o cinema. O Universo amplo da produção audiovisual se introduz na dinâmica da geração caracterizada pela velocidade e dinamismo e ao mesmo tempo a subverte, pois articula inúmeras funções e etapas em um processo lento, progressivo e 
carregado de significados. Entre as etapas da pré-produção e pós-produção, a ideia original de quem cria se modifica, articulando-se com influências, novas descobertas e mudanças de foco. A arte, como se ganhasse vida, conduz o artista ao resultado e o estudante se projeta como sujeito do conhecimento (BARCELOS, 2010).

Win Wenders, diretor alemão de relevância no cinema americano dos anos 80, afirma, ao debater o interesse dos jovens pelo cinema, que a surpresa da juventude é que ela sempre se entrega ao desafio e nesse raciocínio um diretor sempre é aquele que tem algo a contar e de certa forma inovar. Da mesma forma, o cineasta americano David Lynch defende que na criação cinematográfica os obstáculos são inúmeros, mas cada elemento leva a um avanço progressivo que instiga o criador a permanecer no propósito da criação (TIRARD, 2006).

\footnotetext{
Aparentemente, um filme é a transposição de uma ideia em imagem. Mas secretamente, porém, para mim, sempre foi uma maneira de explorar algo de mais pessoal e de mais abstrato. E meus filmes são sempre muito diferentes no final daquilo que eu havia imaginado no início. É um processo evolutivo, portanto. Com frequência, comparo um filme a um navio pirata. É impossível saber aonde o navio vai chegar quando o deixamos livre para seguir os ventos da criatividade. (TIRARD, 2006, p. 157).
}

Dessa forma, podemos inferir que a incorporação da produção em audiovisual digitalrelacionada a conteúdos tradicionais do ensino médio, como o estudo da História, provocaria uma alteração da dinâmica de incorporação desses mesmos conteúdos na construção da cidadania e uma rearticulação no processo de ensino-aprendizagem. Buscando uma melhor compreensão dessas transformações, qualquer estudo que busque uma análise de tais mecanismos e estratégias merece atenção.

\section{Objetivos}

O projeto tem como objetivo principal investigar uma proposta metodológica para o estudo de História no Ensino Médio, a partir da criação de audiovisual digital por meio da elaboração de curtas metragens. Para tanto, estabelecemos como objetivos específicos:

a) Pesquisar as principais dificuldades no ensino e aprendizado de História.

b) Apresentar os principais paradigmas do ensino de História frente às propostas dos Parâmetros Curriculares Nacionais (PCN's).

c) Dialogar sobre a importância do estudo de História no desenvolvimento da cidadania, do senso crítico e da transformação da realidade social. 
d) Investigar e propor possibilidades de utilização da linguagem do cinema digital na elaboração de projetos que contribuam para a compreensão do estudo de História.

e) Investigar o significado da experiência vivenciada em projetos educacionais integrados à linguagem audiovisual digital.

f) Investigar a aprendizagem de História em projetos educacionais associados à criação artística.

g) Propor alternativas para a formação docente nos códigos da linguagem audiovisual.

\section{JUSTIFICATIVA E REFERENCIAL TEÓRICO}

Uma ciência sempre nos parece incompleta se não nos ajuda de alguma forma a viver melhor (BLOCH, 2001) e é inegável que o estudo de História no Ensino Médio recebe dos estudantes uma resistência justamente centrada na ausência de significado. Ecoando no imaginário social, fruto de uma evolução desse conteúdo que ora era ufanista demais, ora controlada peloespectro da censura do autoritarismo pós-64, a disciplina de História sempre esbarrou no utilitarismo da lógica tecnoburocrática (BRASIL, 1997).

Vivemos um novo tempo em que as tecnologias são incorporadas no cotidiano das práticas escolares e a História e seus mecanismos devem se rearticular. Logo, esta ciência que não é só uma ciência em marcha, mas uma ciência na infância (BLOCH, 2001) deve repensar seus caminhos na construção de sua meta fundamental: $\mathrm{O}$ desenvolvimento do aprender $a$ conhecer, aprender a fazer e aprender a conviverna dualidade "eu" e o "outro"(BRASIL, 1997). Nesse sentido, é mister que novas propostas sejam construídas e o ensino evolua na interdisciplinaridade que aproxima a dinâmica do ensino de arte e datecnologia aquiespecificamente o cinema digital - aoensino de História, fundamento do trabalho aqui proposto.

Ao pensar a educação pelo par Experiência/ Sentido, Bondia (2002, p.21) afirma, analisando o significado de cada termo do binômio, que a experiência é o que "nos passa" e não o que "se passa". Percebemos que a apropriação do ocorrido pela visão do indivíduo é o que dá sentido ao evento. A construção desse sentido na experiência é um dos fundamentos da proposta de introdução da linguagem audiovisual na educação tradicional. Ao produzir um curta metragem os indivíduos envolvidos externam suas visões perante a experiência do 
aprendizado.Pensamos que a era da informação somente se configura em era do conhecimento na medida em que a experiência configura o universo àquele que vivencia.

No contexto da realidade brasileira contemporânea, a produção de curtas metragens utilizando a tecnologia digital é uma possibilidade concreta em relação ao ensino. A propagação e a popularização das mídias digitais, o acesso aos canais e programas de edição pela internet e a velocidade da informação nas redes sociais dinamizaram a realidade desses "novos alunos" (PINO \&; ZUIN, 2012). Como é ampla e acessível, já que pode-se realizar um filme com imagens captadas de um celular, a produção de cinema digital proporciona atividades que podem englobar todos os alunos de uma turma, em etapas distintas do processo criativo. Trata-se de uma realidade que deve ser utilizada pelo educador, desde que este introduza os códigos básicos dessa linguagem em projetos de curta, média e longa duração, pois "para nos apropriarmos de uma linguagem, entendermos, interpretarmos e darmossentido a ela, é preciso que aprendamos a operar com seus códigos" (MARTINS, 1998.p.14).

A especificidade do estudo de História na abordagem desse projeto se explica pela importância desta disciplina no repensar e na construção de novos sujeitos histórico-sociais capazes de se posicionaremcriticamente frente a "era da informação" na produção de conhecimento, reagindo à cultura de massas e ao perfil efêmero da contemporânea modernidade líquida (BAUMAN, 2001). Nenhum outro campo do conhecimento evidência tão significativamente o poder das permanências culturais, políticas e econômicas na construção da identidade.

\footnotetext{
Se a primeira premissa fundamental da história é que ela é criada pelo homem, a segunda premissa igualmente fundamental é a necessidade de que nesta criação exista uma continuidade. A história só é possível quando o homem não começa sempre de novo e do princípio, mas se liga ao trabalho e aos resultados obtidos pelas gerações precedentes. Se a humanidade começasse sempre do princípio e se toda ação fosse destituída de pressupostos, a humanidade não avançaria um passo e sua existência se escoaria no círculo da periódica repetição de um início absoluto e de um fim absoluto (KOSIK, 1976, p. 218, grifo do autor).
}

Diversos são os autores e teorias que vão ao encontro das intenções de pesquisa elencadas no projeto. O conceito de alfabetização crítica da mídia, apresentado por Douglas Kellner e Jeff Share em Educação para a leitura crítica da mídia, Democracia Radical e Reconstrução da Educação, foi uma das primeiras questões levantadas durante a elaboração do projeto. A utilização dos recursos de mídia só potencializa o aprendizado se fundamentado em um letramento midiático que permita uma evolução ampla dos envolvidos. (PIMENTA \& 
PRATA-LINHARES, 2013). Tal fluxo de mídia, que coloca identidades e informações de forma inédita (CARRINGTON, 2005), representa terreno fértil para práticas de ensino que dialoguem com as inovações da contemporaneidade. Faz-se necessária uma nova estrutura epistemológica para a alfabetização crítica da mídia, devido ao rápido desenvolvimento da tecnologia da informação e da comunicação e à expansão do capitalismo global de livre mercado. (KELNNER \& SHARE, 2008).

Ainda no sentido de destacar a sociedade da velocidade e as características dos mecanismos de mídia e comunicação, Jenkins (2006) em sua definição de "Cultura de Convergência" apresenta a noção de que as novas tecnologias de mídia dinamizam a criação, dispersão, partilha de conteúdos de mídia mais diversificados e democráticos. A hipótese da intenção de pesquisa, a dinamização do ensino pelas estratégias democráticas de produção em audiovisual, encontra seu fundamento, pois, "precisamos reavaliar os objetivos da educação midiática, para que os jovens possam se ver como produtores culturais e participantes, e não simplesmente como consumidores críticos ou não"(2006, p.259).

\section{PROCEDIMENTOS METODOLÓGICOS}

O Trabalho será realizado em três etapas. A primeira etapa consiste na realização de um levantamento bibliográfico a respeito da temática abordada no projeto, assim como elaboração de uma pesquisa do gênero "estado da arte" sobre as mais recentes publicações a respeito da utilização do audiovisual na educação tradicional.

$\mathrm{Na}$ segunda etapa, serão colhidos e analisados depoimentos dos envolvidos em dois projetos em audiovisual, de autoria e direção próprias, já realizados em uma escola da rede privada da cidade de Uberaba:

a) “A Margem”, Curta Metragem digital realizado por professores no ano de 2009 na cidade de Água Comprida - MG, o qual aborda a situação de exploração do trabalhador rural brasileiro. O projeto, que culminou no referido curta metragem, nasceu de uma iniciativa dos professores de diversas disciplinas com o intuito de inspirar os alunos a realizarem produções audiovisuais que dialogassem com os conteúdos tradicionais. No caso do enredo de "A Margem", o tema de exclusão social no campo engloba temáticas que dialogam com conhecimentos da História, Geografia, Sociologia dentre outros. 
b) "Sete Guerreiros e Uma Lembrança", curta metragem digital realizado por alunos no ano de 2010 na cidade de Uberaba - MG, que aborda uma adaptação da história de Antonio Conselheiro e do arraial de Canudos. A produção, que representa uma continuidade do primeiro projeto, foi realizado por alunos do segundo ano do Ensino Médio e com a participação de três professores, dois dos quais participaram do projeto "A Margem". No enredo do curta metragem, os alunos foram chamados a adaptar a história da guerra de canudos. Vale destacar que, como os estudantes tinham em média 16 anos, foi criada uma história fictícia que envolvia a História da Canudos como estratégia para que eles pudessem participar das filmagens sem quebrar a verossimilhança da história.

Nessa etapa do trabalho serão reunidas, por meio da realização de grupos focais e entrevistas, as percepções atuais dos professores e alunos que se envolveram nos dois projetos a fim de perceber as permanências e leituras dos mesmos a respeito da experiência vivenciada na produção dos curtas metragens. Somados, os 45 indivíduos envolvidos nos dois projetos constituem-se como sujeitos da pesquisa aqui proposta. Com o intuito de englobar todas as esferas do projeto aqui apresentado na dinâmica da produção em audiovisual, tais depoimentos serão filmados e transformados em um documentário de curta metragem durante a realização da pesquisa.

Passaremos então à terceira etapa em que os dados serão analisados e sistematizados a partir dos teóricos citados anteriormente para um estudo dos significados da experiência vivenciada pelos sujeitos da pesquisa.

\section{RESULTADOS E DISCUSSÕES}

A pesquisa encontra-se na etapa de levantamento bibliográfico. Contudo, já foram estabelecidos os contatos com os sujeitos da pesquisa a fim de organizar um cronograma para a realização dos encontros, nos quais serão feitos os grupos focais e entrevistas. Foi elaborado também um artigo do gênero "estado da arte" com o intuito de realizar um levantamento dos trabalhos que debatem a introdução das ferramentas audiovisuais na realidade escolar. Utilizando o banco de teses e dissertações do portal de periódicos da Coordenação de Aperfeiçoamento de Pessoal de Nível Superior, do Ministério da Educação (CAPES/MEC), o artigo aponta a relevância do tema e as múltiplas possibilidades de exploração da linguagem 
de produção de curtas metragens na dinamização de disciplinas tradicionais do ensino básico, como a disciplina de História.

Nos trabalhos selecionados, outro elemento que se evidencia é o fato de que são publicações associadas a Programas de Pós-graduação de áreas diversas como a Psicologia, Ciência da Arte, Comunicação, Linguística e Educação. Fica evidente que o debate acerca das possibilidades da tecnologia na dinamização do aprendizado e na potencialização da experiência ainda está predominantemente fora dos programas ligados à formação de professores. Este receio de absorção da tecnologia como ferramenta real de transformação no universo da escola se relaciona com o fato de o ambiente escolar ser um local onde convivem indivíduos ligados a formações sociais e gerações diferentes. Enquanto alguns professores ainda transitam para a realidades das TIC's, os estudantes absorvem naturalmente a virtualização das relações sociais.

\footnotetext{
As gerações nascidas nas últimas décadas cresceram com a internet, videogames, $C d s$, vídeos, celulares, etc. Estas tecnologias já estavam aqui quando eles nasceram e por eles foram incorporadas com naturalidade, da mesma forma como o fizeram as gerações anteriores com os carros e TVs. Este fato implica, não somente que esta geração tenha total familiaridade com as tecnologias digitais, "daí a denominação nativos digitais" como também, baseando-se em estudos das neurociências, sua forma de pensar, e mesmo a estrutura física de seu cérebro, é diferente das dos imigrantes digitais, que aprenderam e se formaram num mundo analógico e para os quais o mundo digital supõe um processo de imersão nas tecnologias. (PEÑALÓPEZ, 2007 apud SALLES, 2007, p. 7)
}

Portanto, é importante que tais contribuições sejam cada vez mais incorporadas pelas discussões em Educação, instigando novas propostas e caminhos para a subversão do tradicionalismo educacional.

\section{CONSIDERAÇÕES FINAIS}

É evidente que a educação, de uma forma geral, necessita de reflexões que possam reconduzi-la à construção de sentido perante seus sujeitos. As fórmulas tradicionais se esgotaram em uma sociedade de constantes mudanças e a persistência de sistemas tradicionais de ensino, em que o estudante é lançado unicamente à condição de espectador, não direciona as necessidades desse novo mundo. 
As análises dos trabalhos elencados nesta pesquisa serviram sobremaneira para a construção de um referencial teórico que direcionará a intenção de pesquisa aqui apresentada, transformando-a também em uma contribuição para a progressiva superação dos sistemas e metodologias que impedem a articulação entre escola e sociedade, que deve ser, essencialmente, dialética, crítica e ativa.

Ao analisar também as experiências dos sujeitos que vivenciaram a produção dos curtas metragens nos anos de 2009 e 2010, o projeto pretende problematizar a experiência criadora e seus efeitos na longa duração. Observar em que medida o cotidiano dos professores e alunos envolvidos nos eventos se modificou e a visão que os mesmos construíram a respeito da experiência de criação audiovisual articulada ao ensino e à aprendizagem é um dos pontos centrais da pesquisa aqui proposta.

A educação brasileira se mantém entre as mais defasadas em vários estudos comparativos, justamente por persistir em um modelo que remonta ao tradicionalismo de tempos superados, onde professores são os únicos detentores do conhecimento e responsáveis por moldar mentes. Os estudos que se voltam às potencialidades dos mecanismos de comunicação e tecnologia são essenciais à reforma de nosso sistema educacional, e é nesse sentido que caminham as discussões sobre a inclusão das ferramentas do audiovisual na produção e reflexão acerca do conhecimento dentro da escola.

\section{REFERÊNCIAS}

BARCELOS, P. Cinema Educação e Narrativa: esboço para um voo de aeroplano. Dissertação de Mestrado. Universidade de Brasília, Faculdade de Educação. Brasília. 141f. 2010.

BAUMAN, Z. Modernidade Líquida. Tradução Plínio Netizien. - Rio de Janeiro: Zahar, 2001.

BONDIA, J. L. Notas sobre a experiência e o saber de experiência. Rio de Janeiro: Rev. Bras. Educ., n. 19, Apr. 2002 . p. 20-28.

BLOCH, M. L. B. Apologia da História ou o ofício de historiador. Rio de janeiro: Ed. Jorge Zahar. 2001.

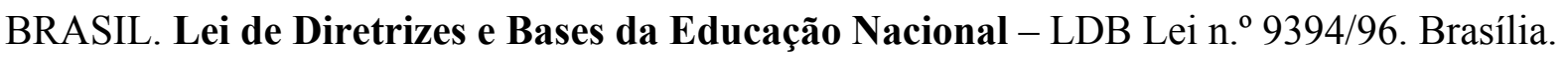

BRASIL. Ministério da Educação e Cultura. Secretaria de Ensino Fundamental. Parâmetros Curriculares Nacionais: História. Brasília: MEC, 1997. 
CARRINGTON, V. New textual landscapes, informationandearlyliteracy. In: Marsh, J. (Ed.). Popular culture, new media and digital literacy in early childhood. London: Routledge Falmer. p.13-17. 2005.

GABRIEL, M. Educar: a (r)evolução digital na educação. São Paulo: Saraiva, 2013.

JENKINS, H. Convergence culture: Where old and new media collide. New York: New York University, 2006.

JESUS, D. C. S. O Programa Mídias na Educação: relações de aproximação e distanciamento entre o trabalho docente e a mídia. Marília, 2011.

KELLNER, D; SHARE, J. Educação para a Leitura Crítica da Mídia, Democracia radical e Reconstrução da Educação. Educ. Soc., Campinas, v.29, n.104 - Especial. 2008 p.687-715.

KOSIK, K. Dialética do concreto. Rio de Janeiro: Paz e Terra. 1976.

MARTINS, M. C. F. D. Didática do ensino de arte: a língua do mundo: poetizar, fruir e conhecer arte. São Paulo: FTD. 1998.

PIMENTA, M.A.A.; PRATA-LINHARES, M. M. Conhecimento e Consumo: desafios para a educação na era da cultura midiática. Revista e-Curriculum (PUCSP), v. 11, 2013. p. 794812.

PINO, I.; ZUIN, A. A. S. A cultura digital e a formação dos professores: uma questão em debate. Educação \& Sociedade. v. 33, n. 121, p. 967-972 Centro de Estudos Educação e Sociedade. Campinas, Brasil. 2012.

TIRARD, L. Grandes diretores de cinema. Tradução Marcelo Jacques de Moraes. Rio de Janeiro: Nova Fronteira, 2006.

RECEBIDO EM: 10/03/2015

APROVADO PARA PUBLICAÇÃO EM: 29/11/2016 